\title{
Secretory leukocyte proteinase inhibitor is preferentially increased in patients with acute respiratory distress syndrome
}

\author{
J.-M. Sallenave*, S.C. Donnelly*, I.S. Grant ${ }^{+}$, C. Robertson*, J. Gauldie ${ }^{\#}$, C. Haslett*
}

Secretory leukocyte proteinase inhibitor is preferentially increased in patients with acute respiratory distress syndrome. J.-M. Sallenave, S.C. Donnelly, I.S. Grant, C. Robertson, J. Gauldie, C. Haslett. C ERS Journals Ltd 1999.

ABSTRACT: Inappropriate release of proteases from inflammatory and stromal cells can lead to destruction of the lung parenchyma. Antiproteinases such as alpha-1-proteinase inhibitor $\left(\alpha_{1}-\mathrm{Pi}\right)$, secretory leukocyte proteinase inhibitor (SLPI) and elastasespecific inhibitor (elafin) control excess production of human neutrophil elastase.

In the present study, the concentrations of $\alpha_{1}-\mathrm{Pi}$, SLPI and elafin found in bronchoalveolar lavage (BAL) fluid from control subjects, patients at risk of developing acute respiratory distress syndrome (ARDS) and patients with established ARDS were determined.

Levels of all three inhibitors were raised in patients compared with normal subjects. SLPI was increased in the group of patients who were at risk of ARDS and went on to develop the condition, compared with the "at-risk" group who did not progress to ARDS ( $p=0.0083) . \alpha_{1}$-Pi and elafin levels were similar in these two populations. In patients with established ARDS, both $\alpha_{1}-\mathrm{Pi}$ and SLPI levels were significantly increased, compared to patients at risk of ARDS who did $(p=0.0089)$ or $\operatorname{did}$ not $(p=$ 0.0003) progress to ARDS.

The finding of increased antiproteinases shortly before the development of acute respiratory distress syndrome provide further evidence for enhanced inflammation prior to clinical disease.

Eur Respir J 1999; 13: 1029-1036.

\begin{abstract}
*Rayne Laboratory, Dept of Medicine, Edinburgh University Medical School, Edinburgh, UK. ' Intensive Therapy Unit, Western General Hospital, Crewe Road, Edinburgh, UK. "Dept of Accident and Emergency, Royal Infirmary Hospital, Lauriston Place, Edinburgh, UK. "Dept of Pathology, McMaster University, Hamilton, Ontario, Canada.
\end{abstract}

Correspondence: C. Haslett, Rayne Laboratory, Dept of Medicine, Edinburgh University Medical School, Edinburgh, EH8 9AG, UK, Fax: 441316504384

Keywords: Acute respiratory distress syndrome, alpha-1-proteinase inhibitor, antiproteinase, elafin, inflammation, secretory leukocyte proteinase inhibitor

Received: April 171998

Accepted after revision October 91998

This study was supported by MRC Canada, the British Lung Foundation and Chest, Heart and Stroke, Scotland. This work was presented in part at the Annual Meeting of the American Thoracic Society, San Francisco, May 1997.
The acute respiratory distress syndrome (ARDS) represents a catastrophic form of acute lung injury that can be provoked by many diverse insults (e.g. multiple trauma, septicaemia, acute pancreatitis). This syndrome in its early stages presents all the characteristic features of acute lung inflammation, with a predominantly neutrophilic alveolitis [1] and resultant leakage of a protein-rich fluid into the interstitium and alveolar spaces. There is considerable evidence implicating neutrophils in the pathogenesis of ARDS.

Significantly, a potent neutrophil chemotactic factor, interleukin (IL)-8 is found in abundance in the alveolar airspace of ARDS patients, and high levels of IL-8 in the bronchoalveolar lavage (BAL) fluid of patients at high risk of developing ARDS are associated with progression to the full-blown disease $[2,3]$. In addition, investigators have described elevated levels of neutrophil-derived products such as the proteolytic enzymes neutrophil elastase $[1,4]$ and collagenase $[5,6]$ in the alveolar space of patients with established ARDS. These enzymes have been im-

For editorial comments see page 947. plicated as injurious agents in chronic lung diseases such as chronic bronchitis and emphysema in which an imbalance between human neutrophil elastase (HNE) and its physiological inhibitors has been postulated to result in parenchymal damage. Historically, alpha-1-proteinase inhibitor $\left(\alpha_{1}-\mathrm{Pi}\right)$ was regarded as the main physiological inhibitor of HNE, following the discovery of an inherited form of emphysema which develops in families homozygous for the $\mathrm{Z}$ allele of the $\alpha_{1}$-Pi gene [7]. However, more recently, two potent low molecular weight inhibitors of HNE, the secretory leukocyte proteinase inhibitor (SLPI) and the elastase-specific inhibitor (ESI), also called elafin, or skin-derived antileukoprotease (SKALP), have been characterized at both the protein and gene level [8-11]. There is now significant evidence that elafin and SLPI are important antiproteases in the lung, in both health and disease [12-15].

To date, no studies have investigated the presence, importance and activities of $\alpha_{1}$-Pi, SLPI and elafin in ARDS. The aim of this study was, therefore, to assess the potential relative importance of these inhibitors within the lung, both in patients in the early risk period for ARDS and in patients with established disease. 


\section{Materials and methods}

\section{Subjects}

In this study, ARDS was defined using the AmericanEuropean Consensus definition [16]. With regard to multiple organ failure, the extent of organ involvement and severity of organ failure were quantified with a modified Goris organ failure score as described previously [17]. Two patient groups were enrolled, first, a group of patients with conditions associated with a high risk of progression to ARDS ("at-risk" group, $n=24$; table 1), and, second, patients with established ARDS disease $(\mathrm{n}=$ 16; table 2). At-risk patients were enrolled on initial hospital presentation and established ARDS patients were enrolled within $24 \mathrm{~h}$ of diagnosis. All patients were attending the Royal Infirmary or Western General Hospital, Edinburgh. Assays were also performed on eight normal control subjects. Informed consent was obtained from patients, or their relatives or guardians. This study was approved by the Lothian Health Board Ethics Committee.

At-risk patients came from two well-defined patient populations, namely severe multiple trauma who required intubation in the emergency room (MT, $n=16)$ and patients with perforated large bowel undergoing laparotomy (PB, $\mathrm{n}=8$ ), the sampling being performed preoperatively when the patients were under general anaesthesia. From this group, 10 patients subsequently progressed to ARDS (seven MT and three PB). The median age of these patients was 51 yrs (range 21-82 yrs). For MT patients, the estimated median time in minutes from the initiating insult to study enrollment was $120 \mathrm{~min}$ (range 90-240 min). While it is
Table 2. - Patient details for the group with established acute respiratory distress syndrome (ARDS)

\begin{tabular}{lcccc}
\hline $\begin{array}{l}\text { Patient } \\
\text { No. }\end{array}$ & Aetiology & Outcome & MOFS & ${\mathrm{Pa}, \mathrm{O}_{2} / F \mathrm{I}, \mathrm{O}_{2}}$ \\
\hline 1 & & & & \\
2 & Sepsis & Lived & 2 & 24 \\
3 & Sepsis & Lived & 5 & 12.7 \\
4 & Sepsis & Lived & 3 & 16.4 \\
5 & Sepsis & Lived & 7 & 16.5 \\
6 & Sepsis & Died & NA & NA \\
7 & Sepsis & Died & 6 & 13.7 \\
8 & Sepsis & Lived & 2 & 17.8 \\
9 & Sepsis & Lived & 2 & 22 \\
10 & Sepsis & Lived & 6 & 18.2 \\
11 & Sepsis & Died & 9 & 14.2 \\
12 & Sepsis & Died & 8 & 14.8 \\
13 & Sepsis & Died & 6 & 9 \\
14 & Sepsis & Lived & 8 & 10.5 \\
15 & Sepsis & Lived & 5 & 29 \\
16 & Sepsis & Died & 6 & 13 \\
\hline
\end{tabular}

MOFS: multiple organ failure syndrome; $\mathrm{Pa}_{\mathrm{a}} \mathrm{O}_{2}$ : arterial oxygen tension; $F \mathrm{I}_{1} \mathrm{O}_{2}$ : inspiratory oxygen fraction; NA: not available. MOFS and lowest $\mathrm{Pa}, \mathrm{O}_{2} / F \mathrm{I}, \mathrm{O}_{2} \quad(\mathrm{kPa})$ are those attained subsequently during the patients' hospital stay.

more difficult to ascertain the precise onset within the PB patient group, the median time of symptoms prior to study enrollment was approximately $24 \mathrm{~h}$ (range 6-72 h). All of the patients in this latter group satisfied the criteria for systemic inflammatory response syndrome (SIRS) [18]. The mean time between bronchoscopy and development of ARDS in the group of patients at risk of ARDS who developed the disease was $47.5 \mathrm{~h}$ (range 6-96 h).

Table 1. - Patient details for the group at risk for acute respiratory distress syndrome (ARDS)

\begin{tabular}{|c|c|c|c|c|c|c|c|}
\hline $\begin{array}{l}\text { Patient } \\
\text { No. }\end{array}$ & Aetiology & TRS & ISS & $\begin{array}{l}\text { Progressed } \\
\text { to ARDS }\end{array}$ & Outcome & MOFS & $\mathrm{Pa}, \mathrm{O}_{2} / F \mathrm{I}, \mathrm{O}_{2}$ \\
\hline 1 & Trauma & 5.676 & 15 & No & Died & 1 & 17.8 \\
\hline 2 & Trauma & 5.221 & 44 & No & Alive & 2 & 27 \\
\hline 3 & Trauma & 7.108 & 33 & No & Alive & 1 & 18 \\
\hline 4 & Trauma & 7.550 & 24 & No & Alive & 3 & 21 \\
\hline 5 & Trauma & 5.970 & 29 & No & Alive & 1 & 45 \\
\hline 6 & Trauma & 2.047 & 29 & No & Died & 1 & 22.3 \\
\hline 7 & Trauma & 4.740 & 38 & No & Died & 0 & 42 \\
\hline 8 & Trauma & 7.11 & 15 & No & Lived & 1 & 29 \\
\hline 9 & Trauma & 4.44 & 29 & No & Died & 0 & 38 \\
\hline 10 & Perf. bowel & & & No & Alive & 1 & 25 \\
\hline 11 & Perf. bowel & & & No & Died & 2 & 27 \\
\hline 12 & Perf. bowel & & & No & Alive & 0 & 29.5 \\
\hline 13 & Perf. bowel & & & No & Alive & 0 & 25.8 \\
\hline 14 & Perf. bowel & & & No & Alive & 0 & $\mathrm{NA}$ \\
\hline 15 & Trauma & 2.628 & 59 & Yes & Lived & 5 & 9.5 \\
\hline 16 & Trauma & 2.198 & 43 & Yes & Died & 6 & 11 \\
\hline 17 & Trauma & 5.97 & 29 & Yes & Lived & 7 & 7.1 \\
\hline 18 & Trauma & 7.841 & 27 & Yes & Lived & 2 & 24 \\
\hline 19 & Trauma & 7.841 & 45 & Yes & Died & 1 & 22 \\
\hline 20 & Trauma & 7.841 & 33 & Yes & Lived & 1 & 29 \\
\hline 21 & Trauma & 7.841 & 29 & Yes & Lived & 2 & 10.2 \\
\hline 22 & Perf. bowel & & & Yes & Lived & 8 & 10.5 \\
\hline 23 & Perf. bowel & & & Yes & Died & 6 & 8.95 \\
\hline 24 & Perf. bowel & & & Yes & Lived & 5 & 12.7 \\
\hline
\end{tabular}

TRS: Trauma Revised Score; ISS: Injury Severity Score; MOFS: Multiple Organ Failure Score; $P \mathrm{a}, \mathrm{O}_{2}$ : arterial oxygen tension; $F \mathrm{I}, \mathrm{O}_{2}$ : inspiratory oxygen fraction; Perf.: perforated; NA: not available. 
All enrolled patients with established ARDS $(n=16)$ had sepsis as their initiating agent. The group median age was 49 yrs (range 18-75 yrs). The patients' demographic data are summarized in tables 1 and 2.

\section{Bronchoscopy}

A bronchoscopy and BAL were performed on enrolled patients. For the "at-risk" patients these procedures were conducted on initial hospital presentation and, in the case of patients with established ARDS, within $24 \mathrm{~h}$ of the diagnosis being made. The fibreoptic bronchoscope was introduced through an indwelling endotracheal tube. The distal end of the bronchoscope was wedged into the middle lobe. Three $60-\mathrm{mL}$ volumes of saline $(0.9 \%(\mathrm{w} / \mathrm{v})$ sodium chloride solution) were instilled, then gently aspirated immediately. On average, $55 \%$ of instilled fluid was recovered (range 40-81\%). All bronchoscopic procedures were performed by one doctor (S.C. Donnelly). Recovered fluid was stored at $4{ }^{\circ} \mathrm{C}$ until processing within $1 \mathrm{~h}$. After straining through sterile gauze, the fluid was centrifuged at $400 \times g$ at $4{ }^{\circ} \mathrm{C}$ for $10 \mathrm{~min}$ to recover cell-free supernatant. BAL supernatant was respun at $1,000 \times g$ for $10 \mathrm{~min}$ at $4{ }^{\circ} \mathrm{C}$ to remove cellular debris and stored at $-70^{\circ} \mathrm{C}$ until assays were performed. Eight normal control subjects (two smokers, five females, mean age $23 \pm 2$ yrs) also underwent BAL.

Enzyme-linked immunosorbent assay for the measurement of antiproteinases

The concentration of $\alpha_{1}$-Pi was determined as described previously [15]. In brief, the first antibody was a polyclonal rabbit anti-human $\alpha_{1}$-Pi immune serum (Sigma, Poole, Dorset, UK) and the second antibody was a sheep anti-rabbit $\alpha_{1}-\mathrm{Pi}$ conjugated to peroxidase (The Binding Site, Birmingham, UK). The reaction was quantified with the chromogenic substrate 2,2-azino-bis(3-ethylbenzthiazoline-6-sulphonic acid) diammonium salt (Sigma) and the absorbance read at $490 \mathrm{~nm} . \alpha_{1}$-Pi purified from human plasma and of a known concentration was used as a standard.

The concentrations of SLPI and elafin were measured using established enzyme-linked immunosorbent assays (ELISA) as described previously [19]. In brief, anti-elafin and anti-SLPI polyclonal rabbit immunoglogulin $\mathrm{G}(\mathrm{IgG})$ were obtained by protein A chromatography [19]. The secondary antibody was a goat horseradish peroxidaseconjugated anti-rabbit IgG adsorbed with human serum proteins (Sigma). The reaction was developed as described above for $\alpha_{1}-\mathrm{Pi}$. Recombinant human elafin, a gift from J. Fitton (Zeneca Pharmaceuticals, Macclesfield, Cheshire, UK), and recombinant SLPI, a gift from R. Heinzel-Wieland (Grunenthal, Stolberg, Germany), were used as standards.

\section{Measurement of albumin}

The albumin level in BAL was determined by immunonephelometry (Kallestad QM300 nephelometer, Montreal, Quebec, Canada), using anti-human albumin antibodies (Sanofi Diagnostics Pasteur, Montreal, Canada).

\section{Total neutrophil elastase inhibitory activity}

The neutrophil elastase inhibitory activity (EIA) in BAL was assessed as described previously [15]. In brief, $100 \mu \mathrm{L}$ BAL supernatant was added to $10 \mu \mathrm{L}$ (300 ng) of activesite titrated human HNE (Elastin Products Company, Owensville, MO, USA) and incubated at $37^{\circ} \mathrm{C}$ for $15 \mathrm{~min}$. The control consisted of HNE incubated with $100 \mu \mathrm{L}$ saline. The mixtures were incubated with the chromogenic substrate $N$-methoxysuccinyl-Ala-Ala-Pro-Val- $p$-nitroanilide (MeO-SAAPVNA) (Sigma; final concentration $0.5 \mathrm{nM}$ ). After a further $5 \mathrm{~min}$ to allow equilibration, hydrolysis of the substrate was recorded at $410 \mathrm{~nm}$ over a 5min period. The amount of BAL fluid sample required to inhibit HNE completely was determined by extrapolation of the linear part of the inhibitory curve to the abscissa, assuming a 1:1 ratio of HNE to inhibitors known to reside in BAL (elafin, SLPI and $\alpha_{1}-\mathrm{Pi}$ ). With this assumption (these three inhibitors have been demonstrated to form a 1:1 stoichiometric complex with HNE [10]), it is possible to calculate EIA, expressed in nM. This value is independent of the affinities $\left(K_{\mathrm{i}}\right)$ of the different inhibitors with HNE [20].

Sodium dodecyl sulphate-polyacrylamide gel electrophoresis and Western blot analysis

Twenty microlitres of BAL supernatant were analysed by sodium dodecyl sulphate-polyacrylamide gel electrophoresis (SDS-PAGE) as described previously [15]. Western blotting was performed using ImmunobilonNC transfer membranes (Millipore, Bedford, MA, USA). Blocking of nonspecific binding sites was performed overnight at room temperature in phosphate-buffered saline (PBS) containing $0.05 \%$ Tween-20 and 5\% skimmed milk powder. Membranes were then incubated with rabbit anti-elafin or anti-SLPI IgG at a dilution of 1:1,000 in blocking buffer, for $1 \mathrm{~h}$ at room temperature. After a further 20-min incubation with goat anti-rabbit IgG conjugated to peroxidase (1:2,500 dilution; Sigma), the blots were developed by enhanced chemiluminescence, according to the manufacturer's instructions (Dupont, NEN Research Products, Boston, MA, USA). The blots were exposed to Reflection NEF autoradiography film (Dupont).

\section{Statistics}

Inhibitor concentrations and activities from the different groups of subjects are expressed as mean \pm SEM and were analysed using a Kruskal-Wallis (unpaired) test followed by a nonparametric Fisher-like test. Linear correlations were used to measure the degree of association between variables. In all cases, $\mathrm{p}<0.05$ was considered as significant.

\section{Results}

\section{Bronchoalveolar lavage cell differentials}

There was no significant difference in BAL cell differentials between patients at risk of ARDS who subsequently developed the disease and those who did not. Therefore, values from at-risk patients were pooled and included in a single group (table 3 ). The mean total cell 
Table 3. - Bronchoalveolar lavage cell differentials in collected samples from at-risk and established acute respiratory distress syndrome patients

\begin{tabular}{lllrc}
\hline & $\mathrm{N}$ & $\mathrm{M}$ & \multicolumn{1}{c}{$\mathrm{L}$} & $\mathrm{E}$ \\
\hline $\begin{array}{l}\text { At-risk } \\
\begin{array}{l}\text { Established } \\
\text { disease }\end{array}\end{array}$ & $4.0(0-7)$ & $84(66-94)$ & $12.0(2-19)$ & $0(0-2)$ \\
\hline
\end{tabular}

Results are expressed as median percentages, with ranges in parentheses. Patients with established ARDS had a very significantly increased percentage of neutrophils compared with patients at risk of ARDS ( $\mathrm{p}=0.0001)$. N: neutrophils; M: macrophages/monocytes; L: lymphocytes; E: eosinophils.

count in BAL from patients at risk of ARDS was $7.8 \times 10^{6}\left( \pm 2.3 \times 10^{6}\right)$ and $8.6 \times 10^{6}\left( \pm 2.1 \times 10^{6}\right)$ from patients with established disease $(p=0.47)$. Patients with established ARDS had a highly significantly increased percentage of neutrophils compared with at-risk patients $(\mathrm{p}=0.0001$, table 3$)$.

Levels of antiproteinases and albumin in bronchoalveolar lavage fluid

There was no statistically significant difference in levels of albumin between normal subjects $(0.23 \pm 0.09 \mu \mathrm{M})$ and at-risk patients who did not develop ARDS (2.23 \pm 4.84 $\mu \mathrm{M})(\mathrm{p}=0.56)$, although there was a trend towards significance between normal subjects and at-risk patients who did develop ARDS $(2.68 \pm 3.21 \mu \mathrm{M})(\mathrm{p}=0.083)$ and between normal subjects and patients with established ARDS $(4.06 \pm 6.27 \mu \mathrm{M})(\mathrm{p}=0.065)$. This may suggest increased alveolar-capillary permeability in patients progressing to ARDS and in those with established ARDS.

Figure 1 shows that all BAL fluid from patients had significantly increased levels of elafin, SLPI and $\alpha_{1}-\mathrm{Pi}$, compared with normal subjects. When BAL fluid from atrisk patients was examined, BAL from those patients who went on to develop ARDS had higher amounts of SLPI than that from patients who did not ( $\mathrm{p}=0.0083$; fig. 1a). In contrast, elafin and $\alpha_{1}$-Pi levels were not significantly different $(\mathrm{p}=0.38$ and 0.54 , respectively). When BAL fluid from at-risk patients was compared with BAL fluid from patients with established ARDS, SLPI levels were found to be increased in the latter ( $\mathrm{p}=0.0003$; fig. 1a). Similarly, $\alpha_{1}$-Pi levels were significantly increased in BAL from patients with established ARDS compared with at-risk patients who did not develop ARDS ( $\mathrm{p}=0.0089$; fig. 1c). In contrast, elafin levels did not change significantly $(\mathrm{p}=$ 0.08 ; fig. 1b).

Figure 2 shows that the total antigenic level of elastase inhibitors (SLPI, elafin, and $\alpha_{1}-\mathrm{Pi}$ ) was significantly increased in BAL fluid from patient groups than in healthy individuals. Levels were also significantly increased in the samples from patients with established ARDS, compared to the samples from at-risk patients but who did not progress to ARDS ( $\mathrm{p}=0.0033)$.

\section{Elastase inhibitory activity in bronchoalveolar lavage}

The EIA was measured in BAL fluid to assess whether the increase in antigenic levels of elastase inhibitors (EI)

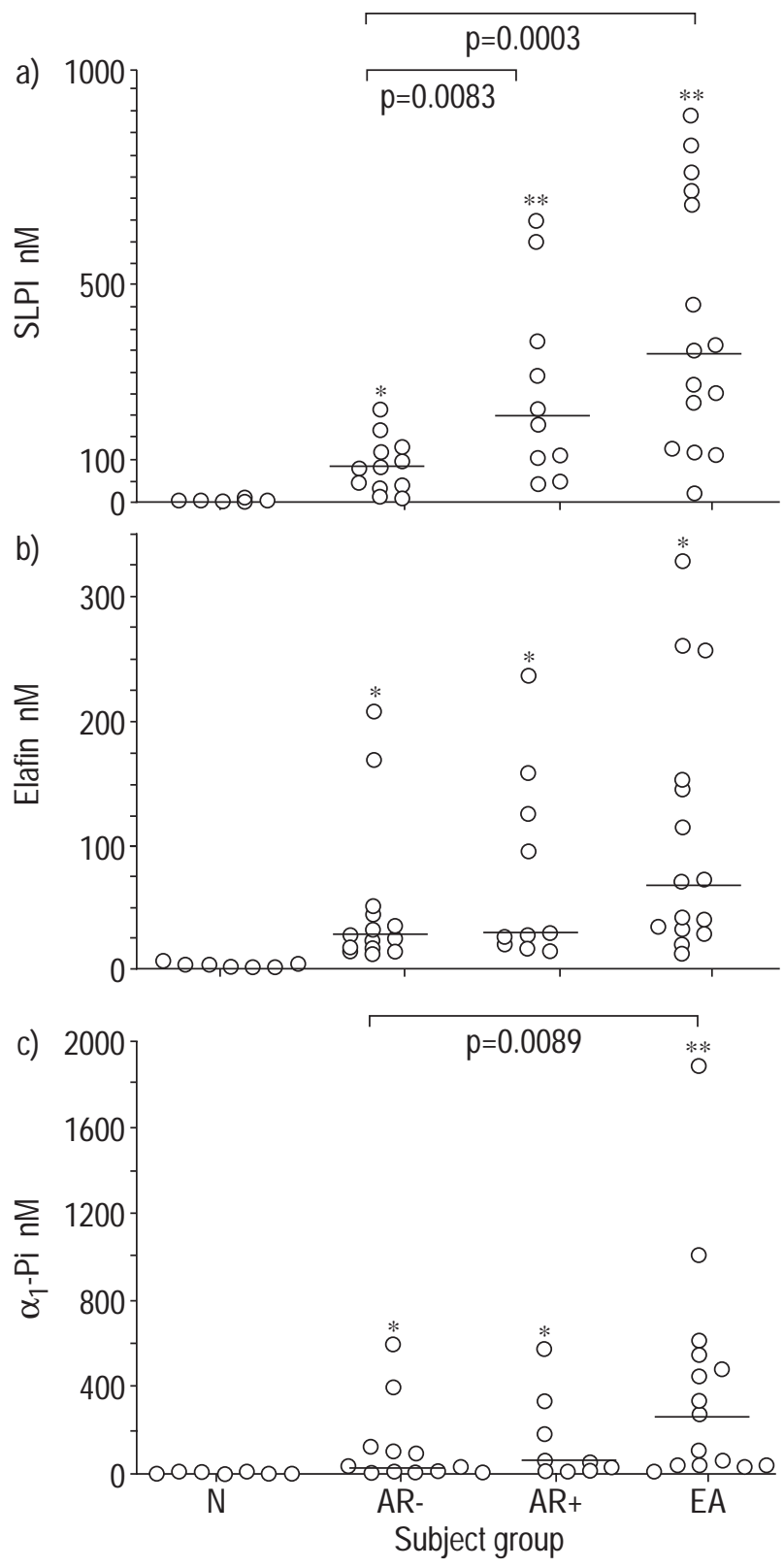

Fig. 1. - Levels of a) secretory leukocyte proteinase inhibitor (SLPI), b) elafin and c) alpha-1-proteinase inhibitor $\left(\alpha_{1}-\mathrm{Pi}\right)$ in bronchoalveolar lavage (BAL) fluid. Individual values of elafin, SLPI and $\alpha_{1}$-Pi levels are shown for the four groups of subjects. N: normal subjects; AR-: atrisk patients who did not develop acute respiratory distress syndrome (ARDS) at a later stage; $\mathrm{AR}+$ : at-risk patients who did develop ARDS at a later stage; EA: patients with established ARDS. Levels were assessed by enzyme-linked immunosorbent assay. *: Significantly different from normal subjects $(\mathrm{p}<0.05)$; **: significantly different from normal subjects and at-risk patients who did not develop ARDS. The median is represented by a horizontal bar.

corresponded with increased activity. In keeping with the ELISA data, all patient groups had increased EIA, compared with the group of normal subjects (fig. 3a). In most of the subjects, the EIA $/ \alpha_{1}$-Pi ratio was $>1$, suggesting that $\alpha_{1}$-Pi was not the only antiprotease active in BAL (fig. 3b).

When compared internally, BAL from the three different groups of patients did not show any significant difference in terms of EIA (fig. 3a), which suggests that in ARDS a 


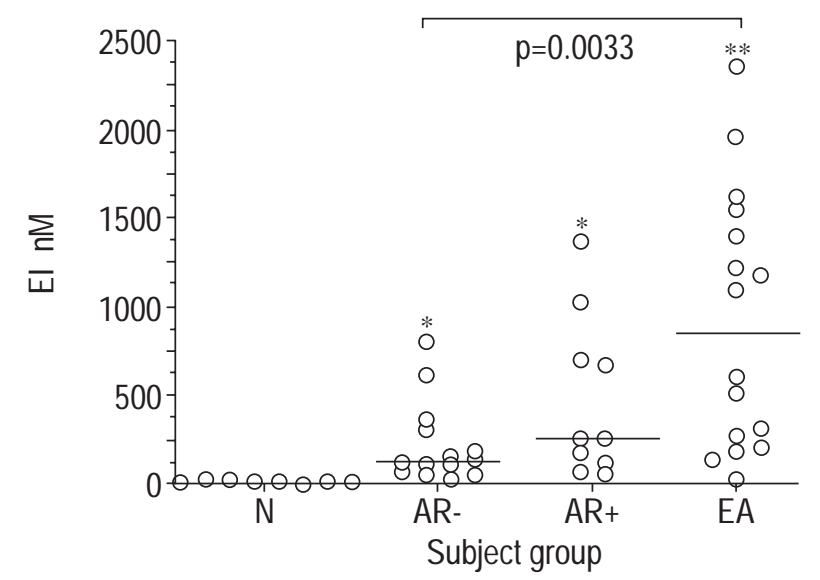

Fig. 2. - Total elastase inhibitor (elafin, secretory leukocyte proteinase inhibitor, and alpha-1-proteinase inhibitor) concentrations (EI) (derived from values presented in fig. 1) in bronchoalveolar lavage fluid from the four groups of subjects: N: normal subjects; AR-: at-risk patients who did not develop acute respiratory distress syndrome (ARDS) at a later stage; $\mathrm{AR}+$ : at-risk patients who did develop ARDS at a later stage; EA: patients with established ARDS. Levels were assessed by enzymelinked immunosorbent assay. *: Significantly different from normal subjects $(\mathrm{p}<0.05) ; * *$ : significantly different from normal subjects and at-risk patients. The median is represented by a horizontal bar.

proportion of the total inhibitors, in particular SLPI and $\alpha_{1}$-Pi (antigen levels of which were significantly increased in BAL fluid from established ARDS patients; figs. 1 and 2), was inactive. In keeping with this, seven out of 16 subjects with established ARDS exhibited an EIA $/ \alpha_{1}$ Pi ratio $<1$ (fig. $3 \mathrm{~b}$ ), suggesting that a considerable proportion of inhibitors was inactive. Indeed, EIA $/ \alpha_{1}-\mathrm{Pi}$ ratios in BAL fluid from patients with established ARDS were significantly lower than in at-risk patients who did not develop ARDS ( $p=0.0012)$. Furthermore, when EIA was corrected for total EI, there was a trend towards a reduction in the values going from normal subjects through to patients with ARDS. The difference became statistically significant when comparing the established ARDS group with normal subjects (fig. $3 \mathrm{c}, \mathrm{p}=0.045$ ).

\section{Western blot analysis}

When BAL from ARDS patients was examined by SDS-PAGE followed by Western blotting (fig. 4) to assess the biochemical characteristics of the three inhibitors studied ( $\alpha_{1}-\mathrm{Pi}$, SLPI and elafin), it was found that $\alpha_{1}$-Pi migrated at the expected location (52 kDa, fig. 4). Similarly, most of the SLPI-immunoreactive species migrated at the expected position $(12 \mathrm{kDa})$, although some material was clearly detectable at a higher position $(55 \mathrm{kDa})$. In contrast, virtually all of the immunoreactive elafin found in ARDS BAL migrated at a higher position $(50 \mathrm{kDa})$ than did recombinant elafin $(10 \mathrm{kDa})$.

\section{Discussion}

Within the lung, an imbalance in favour of excess proteinase has been implicated in such diverse diseases as emphysema, bronchiectasis, cystic fibrosis and ARDS. However, apart from the hereditary form of emphysema in which a link has been established between disease de-
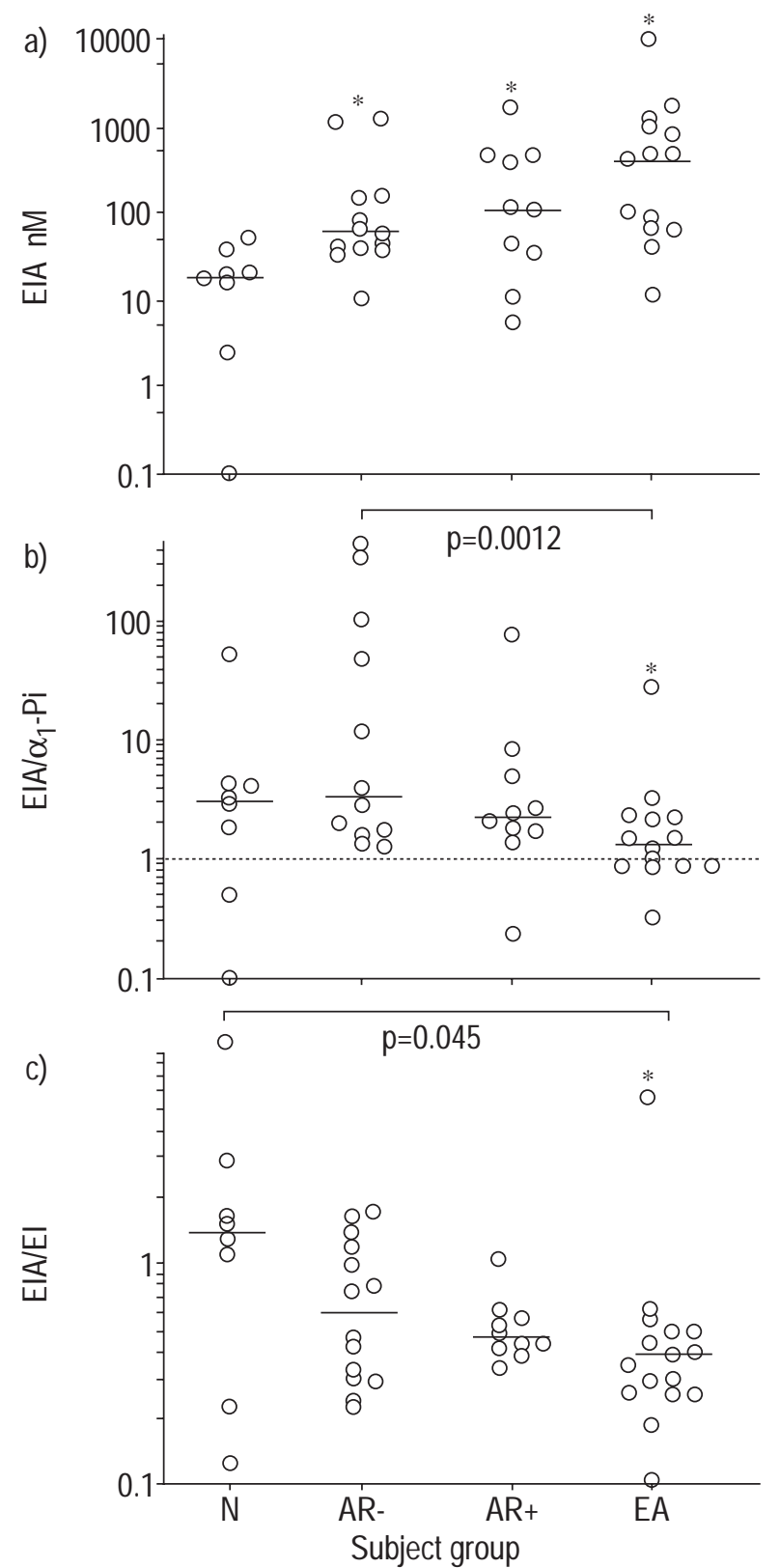

Fig. 3. - Elastase inhibitory activity (EIA) in bronchoalveolar lavage (BAL) from the four groups of subjects: N: normal subjects; AR-: atrisk patients who did not develop acute respiratory distress syndrome (ARDS) at a later stage; $\mathrm{AR}+$ : at-risk patients who did develop ARDS at a later stage; EA: patients with established ARDS. One-hundred microlitres of BAL was added to $10 \mu \mathrm{L}(300 \mathrm{ng})$ of human neutrophil elastase (HNE). Residual HNE activity was measured with MeO-SAAPVNA (see Materials and methods). EIA was also expressed relative to alpha1 -proteinase inhibitor $\left(\alpha_{1}-\mathrm{Pi}\right)$ and relative to total elastase inhibitors (EI). *: Significantly different from normal subjects $(\mathrm{p}<0.05)$. The median is represented by a horizontal bar.

velopment and $\alpha_{1}$-Pi deficiency [7], no conclusive evidence has been produced implicating proteases in the pathogenesis of other inflammatory disease states such as ARDS. In addition, $\alpha_{1}-\mathrm{Pi}$ is not the only elastase inhibitor present in the lung, and low molecular weight elastase inhibitors such as SLPI and elafin are increasingly thought to be important as part of the "antiproteinase shield" in lung defences. 

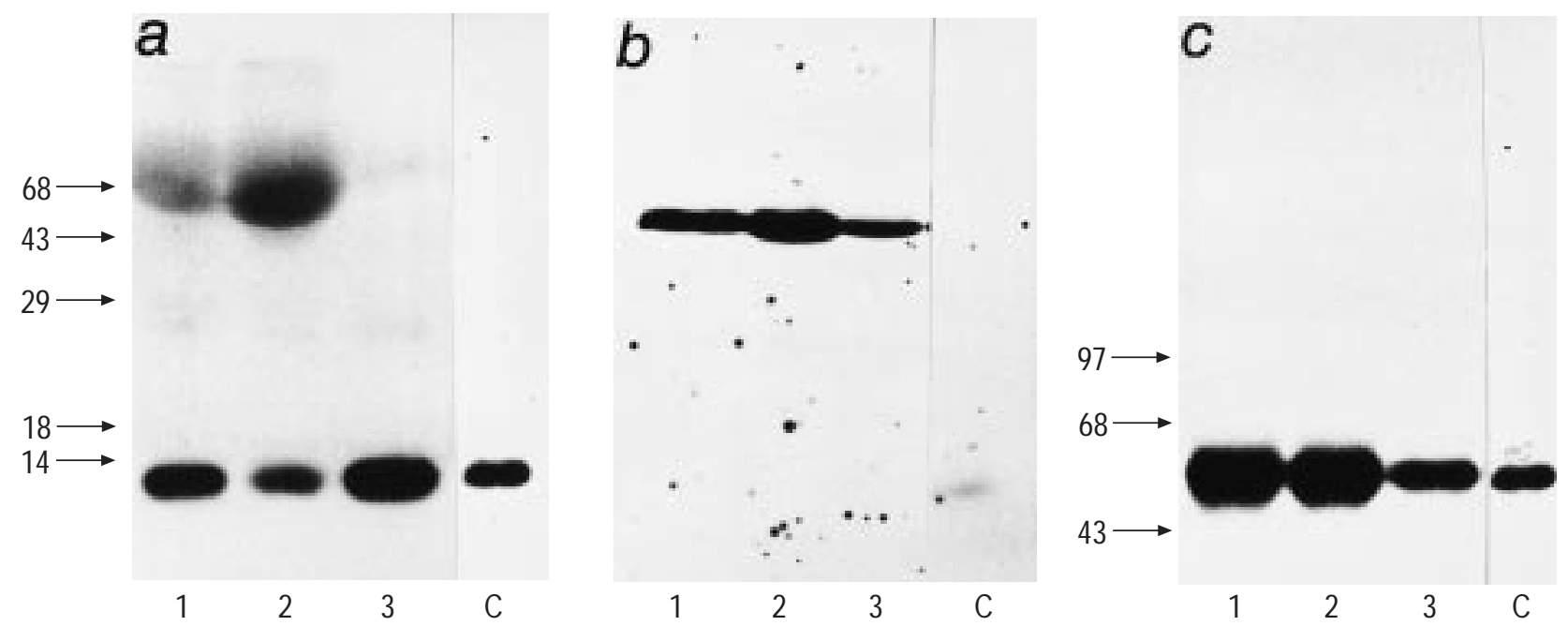

Fig. 4. - Analysis of a) secretory leukocyte proteinase inhibitor (SLPI), b) elafin and c) alpha-1-proteinase inhibitor ( $\alpha_{1}$-Pi) in bronchoalveolar lavage (BAL) fluid samples. Twenty microlitres of BAL samples were loaded and analysed by sodium dodecyl sulphate-polyacrylamide gel electrophoresis followed by Western blot analysis. Lanes 1-3: BAL fluid from three patients with established acute respiratory distress syndrome; lane 4: recombinant SLPI, elafin and purified $\alpha_{1}$-Pi were used as controls. Migration is expressed in kilodaltons, using prestained low molecular weight markers (GibcoBRL) as standards.

Prior to the present study, others have compared BAL from patients with established ARDS and BAL from normal individuals. These studies have suggested that levels of $\alpha_{1}$-Pi [6, 21, 22] and SLPI [21] were increased in established ARDS.

To the authors' knowledge, the present study is the first to assess the intra-alveolar levels of the three major antiproteinases known to inhibit $\operatorname{HNE}\left(\alpha_{1}-\mathrm{Pi}\right.$, SLPI, and elafin), not only in established ARDS, but also at earlier time points in patients at a significant risk of developing the disease.

The main findings of the present study are: 1) the levels of the major antiproteinases were increased not only in established ARDS but also in the at-risk period; 2) the increased levels in the at-risk period provide further evidence for enhanced early inflammation prior to overt clinical disease [3, 20]; and 3) significantly raised levels of SLPI (but not elafin or $\alpha_{1}-\mathrm{Pi}$ ) were found in the alveolar airspaces of at-risk patients who subsequently progressed to ARDS than in those who did not.

Interestingly, it was also found that SLPI levels were highest in patients with established ARDS, in keeping with earlier work by IDELL et al. [21]. This observation held true when SLPI levels were corrected for the number of neutrophils in each patient. However, the state of activation of intra-alveolar neutrophils might be important: activated neutrophils could also be producing SLPI, both in at-risk patients and in patients with established ARDS, consistent with findings in vitro [23].

$\alpha_{1}$-Pi levels were significantly elevated in BAL from patients with established ARDS than in BAL fluid from atrisk patients. Although $\alpha_{1}$-Pi can be secreted locally by inflammatory and structural cells $[24,25]$, most of the $\alpha_{1}$ $\mathrm{Pi}$ reaching the pulmonary airspace is thought to be derived from the circulation. This point was reinforced by the finding that all of the $\alpha_{1}$-Pi recovered in BAL had the same molecular weight as that produced in the liver, which has a different glycosylation pattern and molecular weight from epithelial cell-derived $\alpha_{1}-\mathrm{Pi}[24,25]$ (fig. 4).
Alpha-2-macroglobulin $\left(\alpha_{2}-\mathrm{M}\right)$ is a further elastase inhibitor derived from the circulation (not studied here) which has been found in established ARDS [22]. $\alpha_{2}-\mathrm{M}-$ HNE complexes have residual enzymatic activity against low molecular weight substrates such as MeO-SAAPVNA, which explains why certain studies have found free neutrophil elastase activity in ARDS BAL [22]. However, $\alpha_{2}-\mathrm{M}$ is present at very low concentrations in the BAL and represents $<1 \%$ of the $\alpha_{1}$-Pi molar concentration [22].

When total EI (SLPI, elafin and, $\alpha_{1}-\mathrm{Pi}$ ) were examined, they were found to be significantly increased (compared with normal controls) both in the at-risk period and in established disease. When patient samples were compared internally, levels in the established ARDS group were significantly increased, compared with the at-risk group, reflecting the increase in SLPI and $\alpha_{1}$-Pi in these samples (fig. 2). The present results showing enhanced EIA in all patient samples, compared with normal subjects, confirms previously published work by MAUNDER et al. [26] and Wewers et al. [22]. In most samples, the EIA $/ \alpha_{1}-\mathrm{Pi}$ ratio was $>1$ (fig. 3), clearly showing that $\alpha_{1}-\mathrm{Pi}$ is not the only antiprotease active in BAL. Significantly, the ratio was $>1$ in normal subjects (six out of eight individuals) and in at-risk patients (14 out of 14 patients not developing ARDS and nine out of 10 patients developing ARDS) but was often $<1$ in the established ARDS group (nine out of 16 patients), showing that in the latter group, a number of patients had inactivated $\alpha_{1}$-Pi. When EIA was expressed relative to total inhibitors (EI), the ratio EIA/EI was $>1$ in $75 \%$ of the normal subjects (suggesting the existence of an unidentified elastase inhibitor) and was higher than in patients with established ARDS (fig. 3). However, it was not significantly different between the groups of patients (although there was an obvious trend towards the reduction of the ratio with progression of the disease) (fig. 3). This would suggest that a proportion of these inhibitors are inactivated in ARDS, when the inflammatory process is at an advanced stage, and that ARDS is associated with increased 
inactivation of antiproteases in the alveolar airspace. There are several potential in vivo mechanisms for this observation. Firstly, antiprotease inactivation could result as a consequence of complex formation with HNE. With regard to $\alpha_{1}-\mathrm{Pi}$, Western blot analysis on the collected BAL samples failed to reveal significant quantities of the typical $80 \mathrm{kDa}$ band representing the $\alpha_{1}$-Pi-HNE complex. With regard to SLPI and elafin, complexes with HNE cannot be ruled out since they are not resistant to SDS [27, unpublished data]. A second possibility for antiprotease inactivation is cleavage at or near the active site. For example, in cystic fibrosis, $\alpha_{1}-\mathrm{Pi}$ is cleaved at its active site, generating a $49 \mathrm{kDa}$ inactive protein, migrating more quickly than the native $52 \mathrm{kDa}$ form [28]. Similarly, SLPI can be proteolytically degraded both in vitro and in vivo $[23,29]$. In the present study, it was shown that none of the three inhibitors was cleaved to any significant degree (fig. 4). In contrast elafin and, to a lesser degree, SLPI are complexed to unknown molecules. The complexes have a molecular weight close to $50 \mathrm{kDa}$, similar to one of the complexes found in BAL from farmer's lung disease [15]. Since $\alpha_{1}-\mathrm{Pi}$ cannot account for all of the EIA (see above), and assuming that $\alpha_{1}$-Pi, SLPI and elafin are the only elastase inhibitors in most BAL samples, these data suggest that SLPI and Xelafin complexes are significantly active in these samples. This potentially represents an important observation, as an active X-elafin complex in the interstitium would provide local protection against neutrophil elastase during inflammatory processes, regulating proteinase activity precisely where it is most needed within the target organ.

The overall increase in proteinase inhibitors, and in particular SLPI, in at-risk patients is not associated with protection from the development of overwhelming inflammatory lung injury and ARDS. One explanation for this could be that antiproteases are released as an aborted and unsuccesful attempt to control HNE-driven injury within the inflammatory response.

In conclusion, the present study highlights the importance of secretory leukocyte proteinase inhibitor and other antiproteinases, not only in patients with established acute respiratory distress syndrome, but also in the early "atrisk" period prior to the development of clinically apparent lung injury, at a time when an enhanced intrapulmonary inflammatory response is underway. This is in agreement with in vitro data showing that some of these antiproteinases are under the control of pro-inflammatory cytokines in alveolar cells [19]. The dissection of the regulation of secretory leukocyte proteinase inhibitor and other antiproteinases (such as the tissue inhibitor for metalloproteinase, which has also been found to be increased in acute respiratory distress syndrome) $[30,31]$ secreted at this early time point may offer therapeutic potential in attenuating the pulmonary inflammation in early acute respiratory distress syndrome and reducing morbidity and mortality.

Acknowledgements: The authors are indebted to G. Cunningham, P. Reid, J. Simpson and Z. Xing for reviewing this manuscript.

\section{References}

1. Lee CT, Fein AM, Lippman M, Holtzman H, Kimbel P, Weinbaum G. Elastolytic activity in pulmonary lavage fluid from patients with adult respiratory syndrome. $N$ Engl J Med 1981; 304: 192-196.

2. Donnelly SC, Strieter RM, Kunkel SL, et al. Interleukin-8 (IL-8) and the development of the adult respiratory distress syndrome (ARDS). Lancet 1993; 341: 643-647.

3. Miller EJ, Nagao S, Cohen AB, et al. Elevated levels of NAP-1/interleukin- 8 are present in the airspaces of patients with the adult respiratory distress syndrome and are associated with increased mortality. Am Rev Respir Dis 1992; 146: 427-432.

4. McGuirre WW, Spragg RG, Cohen AB, Cochrane CG. Studies on the pathogenesis of the adult respiratory distress syndrome. J Clin Invest 1981; 69: 543-553.

5. Christner P, Fein A, Goldberg S, Lippman M, Abrams W, Weinbaum G. Collagenase in the lower respiratory tract of patients with adult respiratory distress syndrome. $\mathrm{Am} \mathrm{Rev}$ Dis 1985; 131: 690-695.

6. Weiland JE, Davis WB, Holter JF, Mohammed JR, Dorinsky PM, Gadek JE. Lung neutrophils in the adult respiratory distress syndrome. Am Rev Respir Dis 1986; 133 : 218-225.

7. Laurell CB, Ericksson S. The electrophoretic alpha-1 globulin pattern of serum in alpha-1 antitrypsin deficiency. Scand J Clin Lab Invest 1963; 15: 132-140.

8. Heinzel R, Appelhans RH, Gassen HG, et al. Molecular cloning and expression of cDNA for human antileukoprotease from cervix uterus. Eur J Biochem 1986; 160: 61-67.

9. Wiedow O, Schroder JM, Gregory H, Young JA, Christophers E. Elafin, an elastase-specific inhibitor from human skin. J Biol Chem 1990; 265: 14791-14795.

10. Sallenave J-M, Ryle AP. Purification and characterization of elastase-specific inhibitor. Sequence homology with mucus proteinase inhibitor. Biol Chem Hoppe Seyler 1991; 372: 13-21.

11. Sallenave J-M, Silva A. Characterization and gene sequence of the precursor of elafin, an elastase-specific inhibitor in bronchial secretions. Am J Respir Cell Mol Biol 1993; 8: 439-445.

12. Stockley RA, Morrisson HM, Smith S, Tetley T. Low molecular mass bronchial proteinase inhibitor and alpha-1 proteinase inhibitor in sputum and bronchoalveolar lavage. Physiol Chem Hoppe Seyler 1984; 365: 587-595.

13. Willems LNA, Kramps JA, Stijnen T, Sterk PJ, Weening JJ, Dijkman JH. Antileukoprotease-containing bronchiolar cells. Relationship with morphologic disease of small airways and parenchyma. Am Rev Respir Dis 1989; 139: $1244-1250$

14. Smith SF, Guz A, Cooke NT, Burton GH, Tetley TD. Extracellular elastolytic activity in human lung lavage: a comparative study between smokers and non-smokers. Clin Sci 1985; 69: 17-27.

15. Trembloy G, Sallenave J-M, Israel-Assayag E, Cormier Y, Gauldie J. Elafin/elastase-specific inhibitor in bronchoalveolar lavage of normal subjects and farmer's lung. Am J Respir Crit Care Med 1996; 154: 1092-1098.

16. Bernard GR, Artigas A, Brigham KL, et al. The American-European Consensus conference on ARDS: definitions, mechanisms, relevant outcomes, and clinical trial coordination. Am J Respir Crit Care Med 1994; 149: 818-824.

17. Donnelly SC, Haslett C, Dransfield I, et al. Role of selectins in development of adult respiratory distress syndrome. Lancet 1994; 344: 215-219.

18. Rangel-Frausto MS, Pittet D, Costigan M, Hwang T, Davis CS, Wenzel RP. The natural history of the systemic 
inflammatory response syndrome (SIRS) - a prospective study. J Am Med Assoc 1995; 11: 117-123.

19. Sallenave J-M, Schulmann J, Crossley J, Jordana M, Gauldie J. Regulation of secretory leukocyte proteinase inhibitor (SLPI) and elastase-specific inhibitor (ESI/elafin) in human airway epithelium cells by cytokines and neutrophilic enzymes. Am J Respir Cell Mol Biol 1994; 11: 733-741.

20. Bieth JG. Pathophysiological interpretation of kinetic constants of protease inhibitors. Bull Eur Physiopath Respir 1980; 16: Suppl., 183-195.

21. Idell S, Kucich U, Fein A, et al. Neutrophil elastasereleasing factors in bronchoalveolar lavage from patients with adult respiratory distress syndrome. Am Rev Respir Dis 1985; 132: 1098-1105.

22. Wewers MD, Herzyk DJ, Gadek JE. Alveolar fluid neutrophil elastase activity in the adult respiratory distress syndrome is complexed to alpha-2-macroglobulin. J Clin Invest 1988; 82: 1260-1267.

23. Sallenave J-M, Si-Tahar M, Cox G, Chignard M, Gauldie $\mathrm{J}$. Secretory leukocyte proteinase inhibitor is a major leukocyte elastase inhibitor in human neutrophils. J Leukoc Biol 1997; 61: 695-702.

24. Venembre P, Boutteu A, Seta N, et al. Secretion of alpha1 antitrypsin by alveolar epithelial cells. FEBS Lett 1994; 346: 171-174.

25. Sallenave J-M, Tremblay GM, Gauldie J, Richards C.
Oncostatin M, but not interleukin-6 or leukaemia inhibitory factor, stimulates expression of alpha-1 proteinase inhibitor in A549 human alveolar epithelial cells. $J$ Interf Cytok Res 1997; 17: 337-346.

26. Maunder RJ, Chaney BA, Cokrill DL, Allen KB, Romaneschi LD, Hudson LD. Protease-antiprotease balance favors elastase inhibitors in bronchoalveolar lavage fluid of ARDS patients. Am Rev Respir Dis 1991; 141: A150.

27. Boudier C, Carvallo D, Roitsh C, Bieth J, Courtney M. Purification and characterization of human bronchial proteinase inhibitor. Arch Biochem Biophys 1987; 253: 439445.

28. Goldstein W, Doring G. Lysosomal enzymes from polymorphonuclear leukocytes and proteinase inhibitors in patients with cystic fibrosis. Am Rev Respir Dis 1985; 134: 49-56.

29. Birrer P, McElvaney NG, Rudeberg A, et al. Proteaseantiprotease imbalance in the lungs of children with cystic fibrosis. Am J Respir Crit Care Med 1994; 150: 207-213.

30. Ricou B, Nicod L, Lacraz S, Welgus HG, Suter PM, Dayer JM. Matrix metalloproteinases and TIMP in acute respiratory distress syndrome. Am J Respir Crit Care Med 1996; 154: 346-352.

31. Torii K, Iida K-I, Miyazaki Y, et al. Higher concentrations of matrix metalloproteinases in bronchoalveolar lavage fluid of patients with adult respiratory distress syndrome. Am J Respir Crit Care Med 1997; 155: 43-46. 